\title{
Lower-Complexity Layered Belief-Propagation Decoding of LDPC Codes
}

\author{
Yuan-Mao Chang, Andres I. Vila Casado, Mau-Chung Frank Chang, and Richard D. Wesel \\ Department of Electrical Engineering, University of California, Los Angeles, CA 90095-1594 \\ Email: $\{y m c h a n g$, avila, mfchang, wesel $\} @$ ee.ucla.edu
}

\begin{abstract}
The design of LDPC decoders with low complexity, high throughput, and good performance is a critical task. A well-known strategy is to design structured codes such as quasi-cyclic LDPC (QC-LDPC) that allow partiallyparallel decoders. Also, several works show that sequential schedules, such as Layered Belief-Propagation (LBP), converge faster than the traditional flooding schedule. In this paper, we propose a novel low-complexity sequential schedule called Zigzag LBP (Z-LBP). Current LBP schedules do not allow partially-parallel architectures for some codes, such as high-rate codes with small-to-medium blocklengths. Our proposed algorithm can still be implemented in a partially-parallel manner in these codes. Z-LBP provides the same benefits of LBP such as faster convergence speed and achieves lower frame error rates than flooding.
\end{abstract}

Index Terms - Belief-propagation, channel codes, errorcontrol codes, low-density parity-check codes.

\section{INTRODUCTION}

Low-Density Parity-Check (LDPC) codes, linear block codes defined by a very sparse parity-check matrix $\mathbf{H}$, are often proposed as the channel coding solutions for modern wireless communication systems. Medium-rate LDPC codes are used in standards such as DVB-S2 [1], WiMax (IEEE 802.16e) [2], and wireless LAN (IEEE 802.11n) [3]. Furthermore, high-rate LDPC codes have been selected as the channel coding scheme for mmWave WPAN (IEEE 802.15.3c) [4]. This recent success of LDPC codes is mainly due to structures that allow partially-parallel decoders [5]. These structured codes, called quasi-cyclic LDPC (QCLDPC), are used in all the standards mentioned above.

QC-LDPC codes are represented as an array of submatrices as follows

$$
\mathrm{H}_{Q C}=\left[\begin{array}{ccc}
A_{1,1} & \cdots & A_{1, t} \\
\vdots & & \vdots \\
A_{s, 1} & \cdots & A_{s, t}
\end{array}\right],
$$

where each sub-matrix $A_{i, j}$ is a $p \times p$ circulant matrix. A circulant matrix is a square matrix in which each row is a one-step cyclic shift of the previous row, and the first row is a one-step cyclic shift of the last row.

QC-LDPC decoders have a significantly higher throughput than the decoders of random sparse matrices [6]. The QC-LDPC structure guarantees that at least $p$ messages can be computed in a parallel fashion at all times if flooding schedule is used [5]. Well designed QC-LDPC codes perform as well as random sparse matrices [7].

This work was supported by the US DARPA through the TEAM contract and the Boeing corporation.
The original message-passing schedule, called flooding scheduling, updates all the variable nodes simultaneously using the previously generated check-to-variable messages and then updates all the check nodes simultaneously using the previously generated variable-to-check messages. Sequential message-passing schedules are used to update the nodes sequentially instead of simultaneously. Several studies show that sequential scheduling not only improves the convergence speed in terms of number of iterations but also outperforms the traditional flooding scheduling for a large number of iterations. There are different types of sequential schedules, such as a sequence of check-node updates [8] [9] and a sequence of variable-node updates [10] [11]. Sequential scheduling is also presented in [12] under the name of Layered Belief Propagation (LBP). Similar ideas are presented and analyzed in [13], [14], and [15].

In this paper, we will use the term LBP to denote all sequential schedules. Check-node-centric LBP (C-LBP) indicates a sequence of check-node updates, and variable-nodecentric LBP (V-LBP) indicates a sequence of variable-node updates. Simulations and theoretical results in [8]-[15] show that LBP converges twice as fast as flooding because the messages are updated using the most recent information available as opposed to updating several messages with the same pre-update information. C-LBP has the same decoding complexity per iteration as flooding [9], thus allowing the convergence speed increase at no cost. However, the V-LBP solutions proposed in [10] and [11] have a higher complexity per iteration than flooding and C-LBP. This higher complexity arises from the check-to-variable message computations as will be shown in Section II-B.

Furthermore, QC-LDPC codes where the sub-matrices can have at most one "1" per column and one "1" per row facilitate C-LBP and V-LBP decoding in a partiallyparallel fashion as shown in [9] and [12]. This paritycheck matrix structure allows the partially-parallel processing each of the $p$ nodes over the bi-partite graph, and each processor uses the most recent information available. Thus, QC-LDPC structures guarantee that C-LBP and V-LBP can perform partially-parallel computations and maintain sequential schedule.

However, small-to-medium blocklength high-rate QCLDPC codes generally need more than one diagonal per sub-matrix and only allow one row of sub-matrices. If there were more than one row of sub-matrices, the sub-matrix size will be small, thus diminishing the possible throughputs. Therefore, the C-LBP decoders presented in [9] can- 
not be implemented in a partially-parallel fashion. This issue will be shown in Section IV-A.

We propose a zigzag LBP scheduling scheme called ZLBP that can decode any LDPC code and allows partiallyparallel decoding for QC-LDPC codes. This novel strategy reduces the computation complexity per iteration. Moreover, Z-LBP keeps the advantages of the sequential scheduling such as faster convergence speed and better decoding performance with respect to flooding.

This paper is structured as follows: Section II discusses the issues that arise when implementing flooding and LBP. Section III introduces Z-LBP. Section IV discusses the partially-parallel decoding implementation for high-rate QC-LDPC codes that do not allow C-LBP decoding. Section V delivers the conclusions of this paper.

\section{LBP IMPLEMENTATION ISSUES}

\section{A. Efficient computation of the check-to-variable messages}

The message from check node $c_{i}$ to variable node $v_{j}$ is generated using the following equation,

$$
m_{c_{i} \rightarrow v_{j}}=\prod_{v_{b} \in \mathcal{N}\left(c_{i}\right) \backslash v_{j}} \operatorname{sgn}\left(m_{v_{b} \rightarrow c_{i}}\right) \times \phi\left(\sum_{v_{b} \in \mathcal{N}\left(c_{i}\right) \backslash v_{j}} \phi\left(\left|m_{v_{b} \rightarrow c_{i}}\right|\right)\right),
$$

where $\mathcal{N}\left(c_{i}\right) \backslash v_{j}$ denotes the neighbors of $c_{i}$ excluding $v_{j}$, and $\phi(x)$ is defined as $\phi(x)=-\log \left(\tanh \left(\frac{x}{2}\right)\right) \cdot m_{c_{i} \rightarrow v_{j}}$ is usually generated using a binary operator called Soft-XOR denoted by $\boxplus$

$$
x \boxplus y=\phi(\phi(x)+\phi(y)) .
$$

Soft-XOR is commutative, associative and easy to implement [9] [16] [17]. Eq. (2) can be implemented in practice as follows,

$$
m_{c_{i} \rightarrow v_{j}}=\prod_{v_{b} \in \mathcal{N}\left(c_{i}\right) \backslash v_{j}} \operatorname{sgn}\left(m_{v_{b} \rightarrow c_{i}}\right) \times \bigoplus_{v_{b} \in \mathcal{N}\left(c_{i}\right) \backslash v_{j}} m_{v_{b} \rightarrow c_{i}} .
$$

Eq. (4) shows that $d_{c}-2$ Soft-XORs are required to compute each $m_{c_{i} \rightarrow v_{j}}$. Therefore, $d_{c}\left(d_{c}-2\right)$ Soft-XORs are required to separately compute all the $m_{c_{i} \rightarrow v_{j}}$ from the same check node $c_{i}$.

However, if a message-passing schedule requires the decoder to compute all the $m_{c_{i} \rightarrow v_{j}}$ from the same $c_{i}$ simultaneously, there is an efficient implementation [18]. This efficient implementation is illustrated in Fig. 1. For any degree- $d_{c}$ check node, first generate $d_{c}-2$ intermediate values, $f_{c_{i}, 1}=m_{v_{1} \rightarrow c_{i}}$, and $f_{c_{i}, j}=f_{c_{i}, j-1} \boxplus m_{v_{j} \rightarrow c_{i}}$ for $j=\left\{2, \ldots, d_{c}-1\right\}$. This first step successively accumulates $m_{v_{j} \rightarrow c_{i}}$ in a forward order. Then, generate $d_{c}-2$ intermediate values, $b_{c_{i}, d_{c}}=m_{v_{d_{c}} \rightarrow c_{i}}$, and $b_{c_{i}, j}=b_{c_{i}, j+1} \boxplus m_{v_{j} \rightarrow c_{i}}$ for $j=\left\{d_{c}-1, \ldots, 2\right\}$. This second step successively accumulates $m_{v_{j} \rightarrow c_{i}}$ in a backward order. Finally, $m_{c_{i} \rightarrow v_{j}}$ is computed by doing $f_{c_{i}, j-1} \boxplus b_{c_{i}, j+1}$. This method uses $3\left(d_{c}-2\right)$ Soft-XORs to correctly compute all the $m_{c_{i} \rightarrow v_{j}}$ from the same $c_{i}$ at the same time. This algorithm is optimal in the sense that no algorithm using fewer Soft-XORs

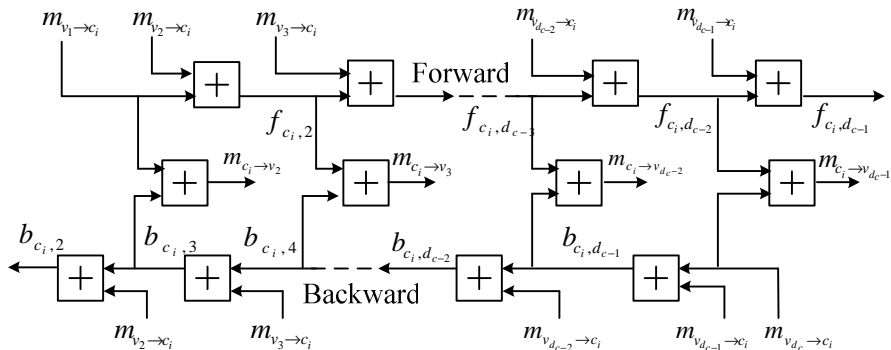

Fig. 1. The most efficient way to calculate $m_{c_{i} \rightarrow v_{j}}$ from the same $c_{i}$

can correctly compute all the $m_{c_{i} \rightarrow v_{j}}$ from the same $c_{i}$ simultaneously. Flooding and C-LBP decoders use this strategy because they compute all the $m_{c_{i} \rightarrow v_{j}}$ from the same $c_{i}$ at the same time.

This check-node update is equivalent to the BCJR algorithm [19] over the trellis representation of the check-node equation in the log-likelihood domain. The forward accumulation of $f_{c_{i}, j}$ corresponds to the BCJR $\alpha$ recursion in the log-likelihood domain. Also, the backward accumulation of $b_{c_{i}, j}$ corresponds to the BCJR $\beta$ recursion in the log-likelihood domain.

\section{B. $V$-LBP implementation issues}

The V-LBP solutions proposed in [10] and [11] have a higher complexity per iteration than flooding and C-LBP which arises from the check-to-variable message computations. Since the V-LBP algorithm sequentially updates variable nodes, it does not allow computing all the $m_{c_{i} \rightarrow v_{j}}$ from the same $c_{i}$ at the same time. Hence, the required number of Soft-XORs to compute all the $m_{c_{i} \rightarrow v_{j}}$ from the same $c_{i}$ is $d_{c}\left(d_{c}-2\right)$.

There is a method, proposed in [14], to reduce the complexity of V-LBP. Define $M_{c_{i}}$ as

$$
M_{c_{i}}=\prod_{v_{b} \in \mathcal{N}\left(c_{i}\right)} \operatorname{sgn}\left(m_{v_{b} \rightarrow c_{i}}\right) \times \bigoplus_{v_{b} \in \mathcal{N}\left(c_{i}\right)} m_{v_{b} \rightarrow c_{i}} .
$$

$M_{c_{i}}$ is the Soft-XOR of all $m_{v_{j} \rightarrow c_{i}}$ destined the same $c_{i}$. A Soft-XOR's inverse operator, Soft-NXOR, denoted by $\boxminus$, is defined as

$$
x \boxminus y=\phi(\phi(x)-\phi(y)) .
$$

Thus, the message from check-node $c_{i}$ to variable-node $v_{j}$ can be computed by

$$
m_{c_{i} \rightarrow v_{j}}=M_{c_{i}} \boxminus m_{v_{j} \rightarrow c_{i}} .
$$

The decoder first initializes all $M_{c_{i}}$ for each check node. Then, separately generates all the $m_{c_{i} \rightarrow v_{j}}$ using Eq. (7). Also, when a new $m_{v_{j} \rightarrow c_{i}}$ is computed, $M_{c_{i}}$ is re-calculated using

$$
M_{c_{i}}=m_{c_{i} \rightarrow v_{j}} \boxplus m_{v_{j} \rightarrow c_{i}} .
$$

In each iteration, computing all the $m_{c_{i} \rightarrow v_{j}}$ from the same $c_{i}$ requires $d_{c}$ Soft-NXORs. Moreover, $d_{c}$ Soft-XORs are needed to re-calculate $M_{c_{i}}$ since there will be $d_{c}$ new $m_{v_{j} \rightarrow c_{i}}$ on every iteration. Assuming that the complexity of Soft-XOR and Soft-NXOR is the same, the number of 
required operations per iteration needed to update a check node is $2 d_{c}$. We omit the $d_{c}$ Soft-XORs required to compute $M_{c_{i}}$ initially in Eq. (5).

However, Soft-XOR is not invertible on every point. Without loss of generality, assume $m_{v_{1} \rightarrow c_{i}}$ is 0 . Then, $M_{c_{i}}$ is 0 , so $m_{c_{i} \rightarrow v_{1}}=\phi(\phi(0)-\phi(0))=\infty$. Also, even if all $\left|m_{v_{j} \rightarrow c_{i}}\right|$ are bigger than 0 , this algorithm is still numerical unstable because the dynamic range of Soft-NXOR is $[0, \infty)$. When the two arguments of Soft-NXOR are similar, the output is very large and out of quantization levels. The large quantization noise makes this strategy not practical for implementation. The authors of [17] also arrived to the conclusion that this technique is not feasible in practice.

\section{ZIGZAG LBP}

We propose a novel LBP schedule that requires fewer number of operations per iteration than flooding, C-LBP, and V-LBP to compute all the $m_{c_{i} \rightarrow v_{j}}$. Zigzag LBP is a V-LBP strategy that performs variable-node updates in a zigzag pattern over the parity-check matrix. One directional updating, forward updating or backward updating of all variable nodes, corresponds to one iteration. Zigzag updating guarantees that all the $m_{c_{i} \rightarrow v_{j}}$ can be generated by using the technique presented in Section II-A.

The Z-LBP algorithm is formally presented in Algorithm 1. First, the decoder initializes all $f_{c_{i}, j}$ of the every check node. Then, the first iteration, as well as all the odd iterations, consists of the sequential update of variable nodes $v_{j}, j=\{N \ldots 1\}$ in a backward fashion. All the $m_{c_{i} \rightarrow v_{j}}$ destined to the same variable node $v_{j}$ are generated using $f_{c_{i}, j-1} \boxplus b_{c_{i}, j+1}$. Then, the decoder generates all the $m_{v_{j} \rightarrow c_{i}}$ from the same $v_{j}$. Finally, the decoder calculates all the $b_{c_{i}, j}$ for every $c_{i}$ that is a neighbor of $v_{j}$ using $b_{c_{i}, j+1} \boxplus m_{v_{j} \rightarrow c_{i}}$. The second iteration, as well as all even iterations, updates the variable nodes $v_{j}, j=\{1 \ldots N\}$ in a forward fashion. All the $m_{c_{i} \rightarrow v_{j}}$ of the same variable node $v_{j}$ are still generated using $f_{c_{i}, j-1} \boxplus b_{c_{i}, j+1}$, and then all the $m_{v_{j} \rightarrow c_{i}}$ are generated. Finally, the decoder calculates all the $f_{c_{i}, j}$ for every $c_{i}$ that is a neighbor of $v_{j}$ using $f_{c_{i}, j-1} \boxplus m_{v_{j} \rightarrow c_{i}}$.

The decoder initializes all the $f_{c_{i}, j}$ in Line 3 of Algorithm 1 following the order of the received channel information. Hence, the decoder simultaneously receives all the channel information and initializes all the $f_{c, j}$. The ZLBP algorithm computes all the $m_{c_{i} \rightarrow v_{j}}$ using the forward and backward technique which is described in Section II-A in a distributed fashion. However, the decoder computes $m_{c_{i} \rightarrow v_{j}}$ and either $f_{c_{i}, j}$ or $b_{c_{i}, j}$ instead of both of them in each iteration. Thus, it requires a fewer number of SoftXORs to update a check node. Z-LBP requires $2\left(d_{c}-2\right)$ Soft-XORs in order to update a check node. Flooding and C-LBP require $3\left(d_{c}-2\right)$ Soft-XORs to update a check node, and V-LBP needs $d_{c}\left(d_{c}-2\right)$ Soft-XORs. Thus, if we assume the complexity of computing check-to-variable messages is much higher than the complexity of computing variableto-check messages [9] [18], Z-LBP is 1.5 times simpler than flooding and C-LBP and $d_{c} / 2$ times simpler than V-LBP per iteration.

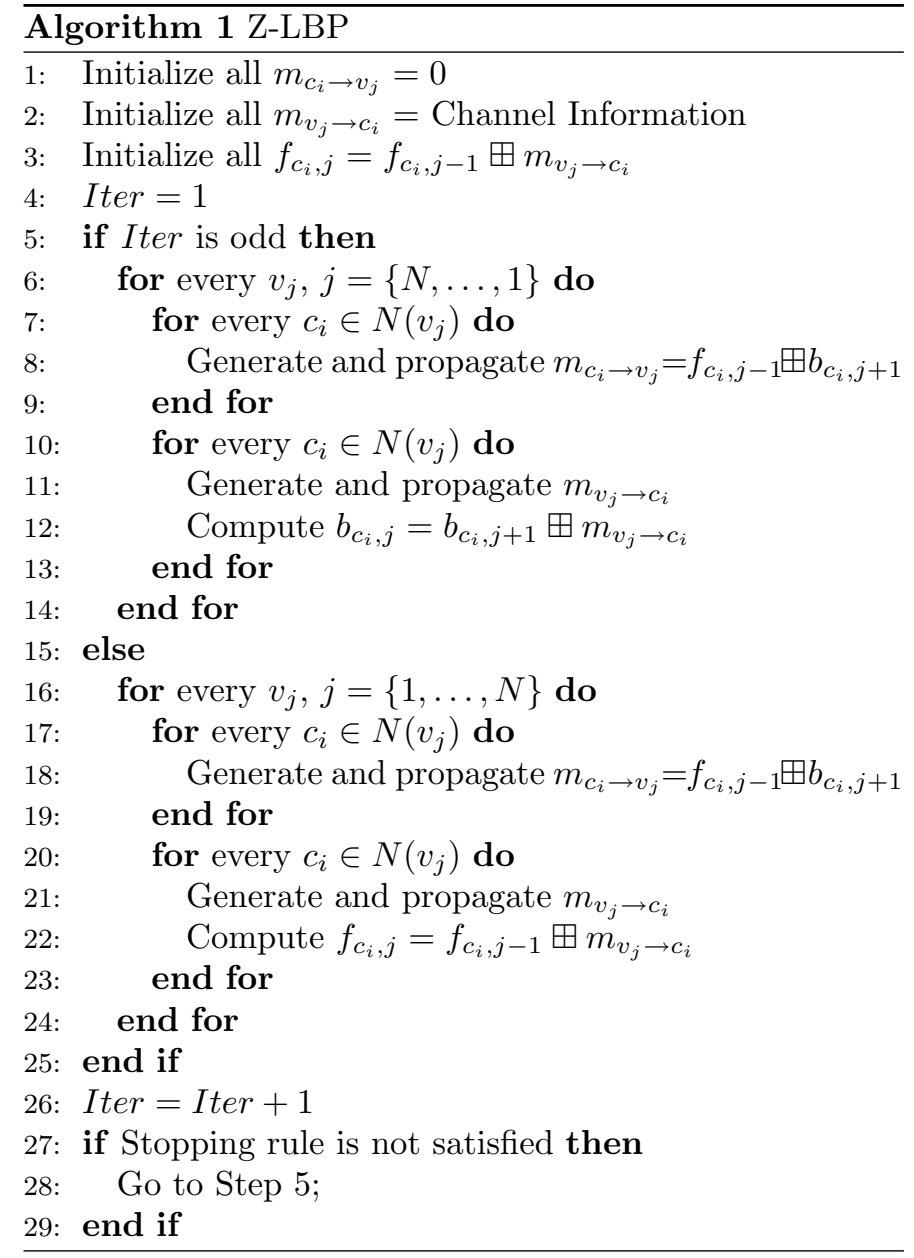

Let us denote the number of the edges of the bi-partite graph as $N_{E}$. There are $N_{E} f_{c, j}$ values and $N_{E} b_{c, j}$ values. This suggests that the Z-LBP decoder needs a memory of size $2 N_{E}$. However, in the case of an odd iteration, the decoder computes a new $b_{c, j}$ after updating $m_{v_{j} \rightarrow c_{i}}$. Thus, the new $b_{c, j}$ can be written in the same memory address of $f_{c, j}$ given that $f_{c, j}$ is not needed anymore. The same can also be said about the even iterations, the new $f_{c, j}$ can be written in the same memory address of $b_{c, j}$. Therefore, the required memory size is only $N_{E}$. This is the same memory size required for a C-LBP decoder which is half the memory required for a flooding decoder [9].

Fig. 2 shows the AWGN performance of four different scheduling strategies, flooding, V-LBP, C-LBP, and Z-LBP as the number of iterations increases. All the simulations correspond to the blocklength-1944 rate-1/2 LDPC code presented in the IEEE 802.11n standard [3]. This figure shows that Z-LBP has a better convergence speed than flooding across all iterations. The frame error rate of flooding around 20 and 40 iterations are equal to the frame error rate of Z-LBP around 15 and 30 iterations respectively. However, since the computation complexity of Z-LBP is 1.5 times simpler than that of flooding, Z-LBP's convergence speed in terms of the number of Soft-XORs is twice as much as that of flooding. C-LBP and V-LBP's convergence speeds in terms of the number of iterations are 


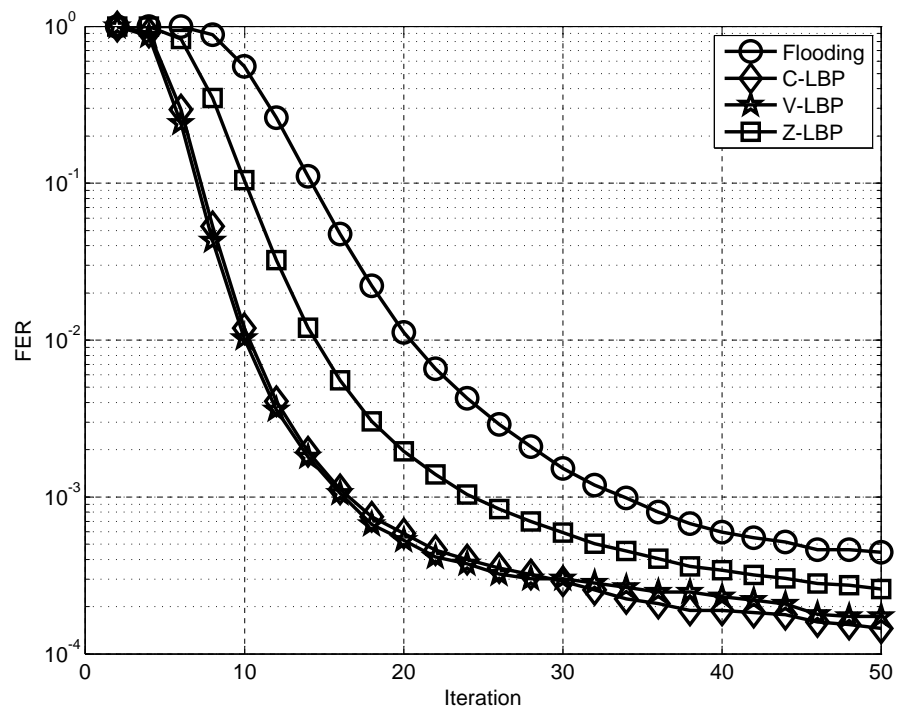

Fig. 2. Performance of flooding, C-LBP, V-LBP, and Z-LBP at different iterations for a fixed $E_{b} / N_{0}=1.75 \mathrm{~dB}$

similar. Although Z-LBP's convergence speed in terms of the number of iterations is around 1.5 slower than that of C-LBP, the computation complexity of Z-LBP is 1.5 times simpler than that of C-LBP per iteration. Thus, C-LBP and Z-LBP's convergence speeds in terms of the number of Soft-XORs are almost the same.

For a degree- $d_{c}$ check node, the computation complexity of Z-LBP is $d_{c} / 2$ times simpler than that of V-LBP. The code in the IEEE $802.11 \mathrm{n}$ standard has the check-node degrees 7 and 8 . Thus, the computation complexity of Z-LBP is 3.5 times simpler than that of V-LBP. Hence, Z-LBP's convergence speed in terms of the number of Soft-XORs is around 2 times faster than V-LBP.

Fig. 3 shows frame error rates of these four scheduling strategies presented above at different SNRs. Since the complexity of Z-LBP is 1.5 times simpler than flooding and C-LBP, the 50-iteration computation complexity of Z$\mathrm{LBP}$ is equivalent to the 33-iteration that of flooding and C-LBP. Similarly, Z-LBP is 3.5 times simpler than V-LBP. Thus, the 50-iteration Z-LBP corresponds to 14-iteration V-LBP. The performance of Z-LBP is $0.15 \mathrm{~dB}$ better than flooding. There is no difference between C-LBP and ZLBP's performance. However, the coding gain between Z$\mathrm{LBP}$ and V-LBP is around $0.2 \mathrm{~dB}$.

\section{IMPLEMENTATION ISSUES FOR MEDIUM BLOCKLENGTH High-RATE LDPC CODES}

Modern wireless communication systems provide higher and higher throughputs. IEEE 802.11a [20] can provide tens of Mbps, and IEEE 802.11n [3] improves the throughput to hundreds of Mbps. Recently, a wireless communication standard, IEEE 802.15.3c [4] targets throughputs on the order of Gbps. Hence, high-rate LDPC codes with high-throughput decoders are needed.

Parity-check matrices of small-to-medium blocklength high-rate QC-LDPC codes have one row of sub-matrices, where each sub-matrix consists of several (more than one)

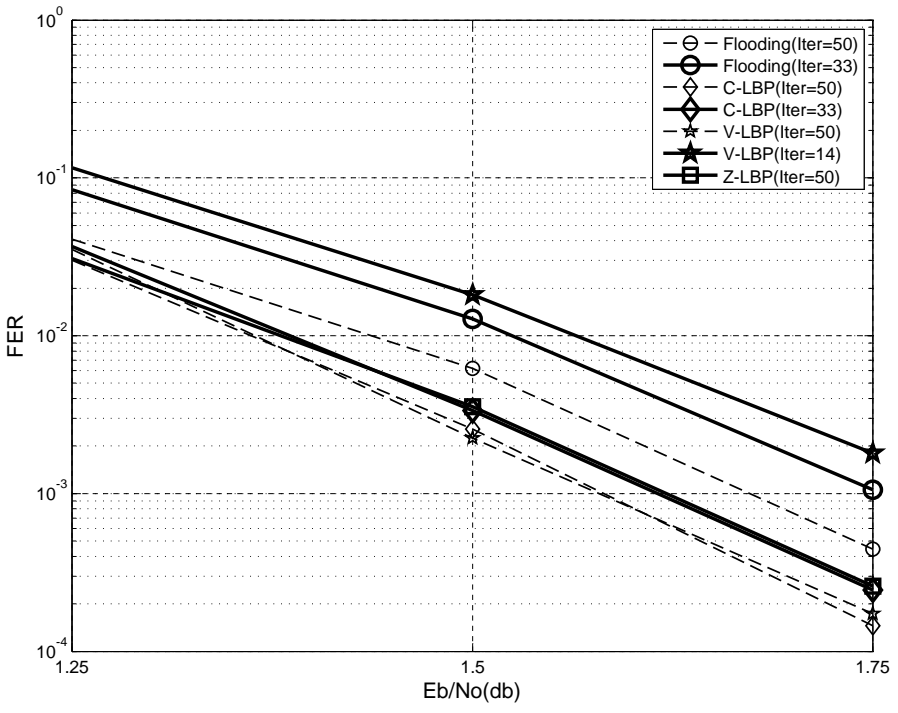

Fig. 3. Frame error rate performance of flooding, C-LBP, V-LBP, and Z-LBP for different iterations v.s. $E_{b} / N_{0}$

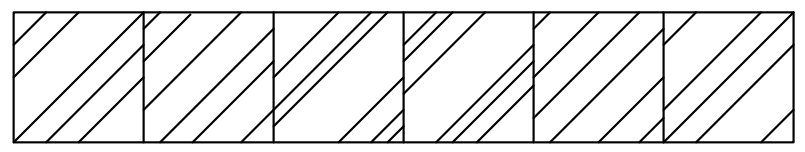

Fig. 4. The structure of a parity check matrix

cyclic-shift diagonals in order to avoid degree- 1 variable nodes. In these cases, the single row of sub-matrices is necessary because multiple rows would require the sub-matrix size to be too small to provide the necessary throughput. Fig. 4 shows the structure of the parity-check matrix of a regular high-rate LDPC code. Diagonal lines represent the "1"s of $\mathbf{H}$. For example, the rate-14/15 LDPC code proposed in the IEEE $802.15 .3 \mathrm{c}$ standard is a regular code with a similar structure to the one shown in Fig. 4. Its blocklength is 1440, and its check-node degree $d_{c}$ is 45 .

\section{A. C-LBP implementation issues}

Algorithm 2 describes the partially-parallel version of the C-LBP algorithm [9]. The C-LBP decoder processes one row of sub-matrices at the same time. Separate processors simultaneously update all check nodes $C_{l}$ in the same row of sub-matrices $l$. Different variable-to-check messages $m_{V \rightarrow C_{l}}$ must be generated and propagated at the same time. If each sub-matrix contains at most one "1" per column and one " 1 " per row, the processors access disjoint sets of variable nodes. This guarantees that each processor uses the most recent information available even if all the processors perform in parallel.

However, for small-to-medium blocklength high-rate QC-LDPC codes, the C-LBP algorithm presented in [9] cannot be implemented in a partially-parallel fashion. The reason is that the parity-check matrix contains only one row of sub-matrices. Thus step 3 and 4 in Algorithm 2 become the variable-node update and check-node update of the flooding scheduling respectively. Therefore, partiallyparallel C-LBP becomes exactly the same as flooding in 


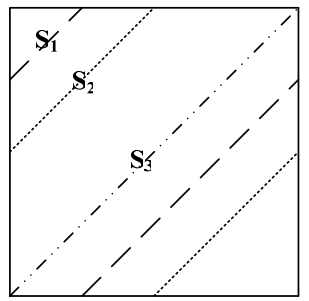

Fig. 5. The labeled cyclic-shift diagonals in one sub-matrix

complexity, convergence speed, and decoding capability. Partially-parallel C-LBP for small-to-medium high-rate QC-LDPC codes is not a sequential schedule.

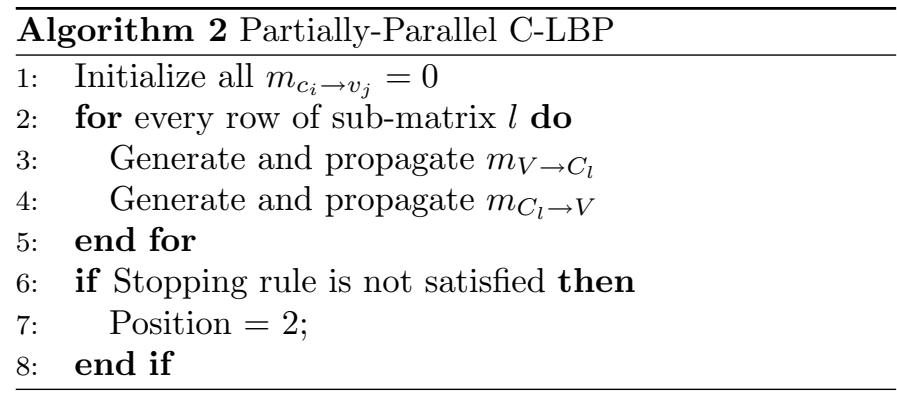

\section{B. Partially-parallel implementation of Z-LBP}

Z-LBP can perform in a partially-parallel fashion by updating a column of sub-matrices. First, label the cyclicshift diagonals in each sub-matrix as shown in Fig. 5. Assume there are $N_{\text {mat }}$ sub-matrices, and each sub-matrix has $N_{\text {diag }}$ cyclic-shift diagonals $\left(N_{\text {diag }}>1\right)$. Then, the order of variable-node updates at step 6 in Algorithm 1 is slightly changed to "for every column of sub-matrix $S M_{j} j=\left\{N_{\text {mat }}, \ldots, 1\right\}$." This labeling prevents memory access conflicts when all processors process $p$ variable nodes at the same time. All the $m_{c_{i} \rightarrow v_{j}}$ are still computed using $f_{c, j-1} \boxplus b_{c, j+1}$. However, since $N_{\text {diag }}>1$, the decoder requires extra $d_{c}-N_{\text {mat }}$ Soft-XORs in order to compute $f_{c_{i}, j}$ or $b_{c_{i}, j}$ in advance. For example, when the decoder prepares to update the sub-matrix $S M_{2}$ in a forward fashion, the decoder needs to compute $f_{c, N_{\text {diag }}+j}$ $j=\left\{1, \ldots, N_{\text {diga }}-1\right\}$ in advance. Because of the computation $f_{c_{i}, j}$ or $b_{c_{i}, j}$ in advance, the decoder does not use the recent information available at step 8 and 18 in Algorithm 1 . However, this does not diminish the performance significantly.

Consider the rate-14/15 QC-LDPC code used in IEEE 802.15.3c. The check-node degree $d_{c}$ is equal to 45 , and there are 15 sub-matrices. Hence, Z-LBP in a partiallyparallel fashion requires 114 Soft-XORs to compute all the $m_{c_{i} \rightarrow v_{j}}$ from the same check node $c_{i}$. V-LBP needs 1935 Soft-XORs to compute all the $m_{c_{i} \rightarrow v_{j}}$ from the same $c_{i}$. The flooding schedule requires 129 Soft-XORs to compute all the $m_{c_{i} \rightarrow v_{j}}$ from the same $c_{i}$. Therefore, Z-LBP is 17 times and 1.13 times simpler than V-LBP and flooding respectively.

Fig. 6 shows the AWGN performance of three different scheduling strategies, flooding, V-LBP, and Z-LBP in

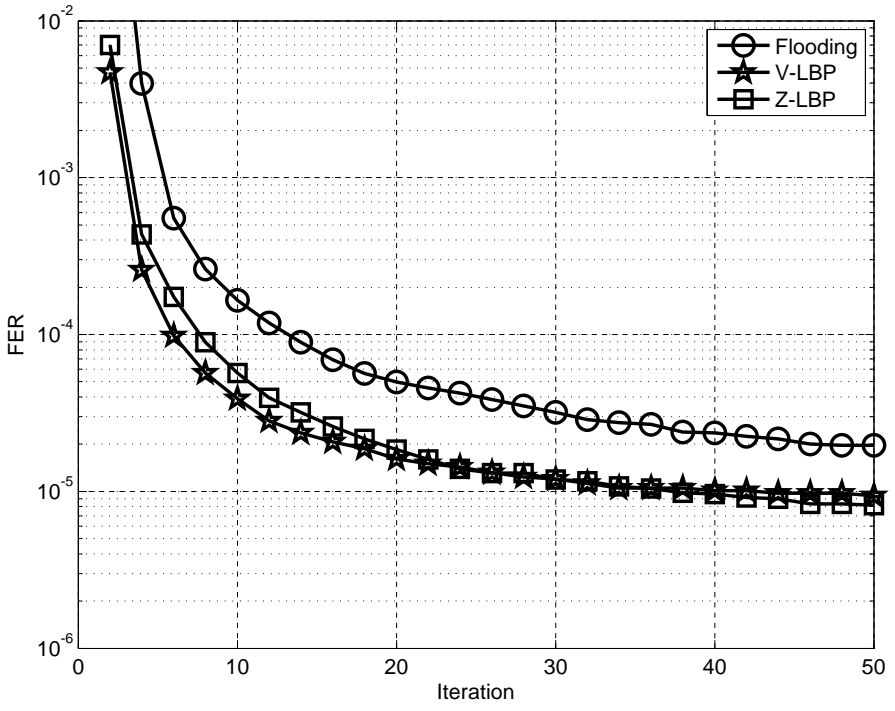

Fig. 6. Performance of flooding, C-LBP, V-LBP, and Z-LBP in a partially-parallel fashion at different iterations for a fixed $E_{b} / N_{0}=6.0 \mathrm{~dB}$

a partially-parallel fashion, as the number of iterations increases. All the simulations use the same blocklength-1440 rate-14/15 LDPC code proposed in the IEEE 802.15.3c standard. The figure shows that Z-LBP in a partiallyparallel fashion has better convergence speed than flooding across all iterations. Z-LBP's convergence speed in terms of the number of Soft-XORs is around 3 times faster than flooding. Moreover, the convergence speed in terms of iterations of Z-LBP and V-LBP are similar. However, Z-LBP is 17 times simpler than V-LBP. Hence, the convergence speed in terms of the number of Soft-XORs of Z-LBP is much faster than that of V-LBP.

Fig. 7 shows frame error rates of these three scheduling strategies presented above in a partially-parallel fashion at different SNRs. Since the complexity of Z-LBP is 17 times and 1.13 times simpler than V-LBP and flooding respectively, the 50-iteration complexity of Z-LBP is equal to 3-iteration V-LBP and 44-iteration flooding. Fig. 7 shows the coding gain's gap between flooding and Z-LBP is $0.125 \mathrm{~dB}$. The performance of Z-LBP is $0.5 \mathrm{~dB}$ better than that of V-LBP.

\section{CONCLUSION}

We propose Z-LBP, a low-complexity sequential schedule of variable node updates. For a degree- $d_{c}$ check-node, the computation complexity per iteration of Z-LBP is $d_{c} / 2$ times simpler than that of V-LBP. Also, Z-LBP is 1.5 times simpler than flooding and C-LBP. Z-LBP outperforms flooding with a faster convergence speed and better decoding capability.

For QC-LDPC codes where the sub-matrices can have at most one "1" per column and one "1" per row, Z-LBP can perform partially-parallel decoding. It provides the same performance as C-LBP. Therefore, Z-LBP is alternative implementation of LBP.

However, for small-to-medium blocklength high-rate 


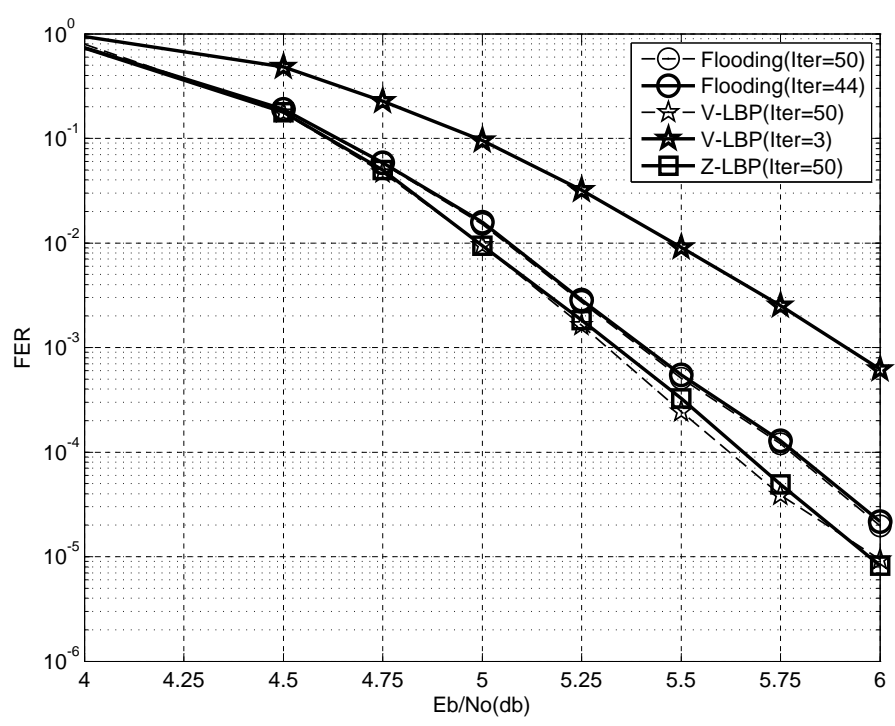

Fig. 7. Frame error rate performance of flooding, V-LBP, and Z-LBP in a partially-parallel fashion at different $E_{b} / N_{0}$

QC-LDPC codes whose parity-check matrix contains only one row of sub-matrices, partially-parallel C-LBP is exactly the same as flooding. In contrast, the proposed Z-LBP can still perform partially-parallel decoding and maintains a sequential schedule.

\section{REFERENCES}

[1] European Telecommunications Standards Institute (ETSI). Digital Video Broadcasting (DVB) Second generation, framing structure for broadband satellite applications; EN 302307 V1.1.1. 2005.

[2] IEEE 802.16e: Air Interface for Fixed and Mobile Broadband Wireless Access Systems, IEEE, 2004.

[3] IEEE P802.11n/D1.05 October 2006, Wireless LAN Medium Access Control (MAC) and Physical Layer (PHY) specifications Enhancements for Higher Throughput (Draft).

[4] IEEE P802.15.3c/July 2007, Wireless Personal Area Network (WPAN) Standard Physical Layer (PHY) specifications (Draft).

[5] M.M. Mansour and N.R. Shanbhag. Turbo decoder architectures for low-density parity-check codes. In Proc. IEEE Global Conference on Communications, pages 1383-1388, Taipei, Taiwan, November 2002

[6] Z. Li, L. Chen, L. Zeng, S. Lin, and W. Fong. Efficient encoding of low-density parity-check codes. IEEE Trans. on Commun., 54:71-81, January 2006.

[7] L. Chen, J. Xu, I. Djurdjevic, and S. Lin. Near Shannon limit quasi-cyclic low-density parity-check codes. IEEE Trans. on Commun., 52(7):1038-1042, July 2004.

[8] E. Yeo and P. Pakzad and B. Nikolic and V. Anantharam. High throughput low-density parity-check decoder architectures. In Proc. 2001 Global Conference on Communications, pages 30193024, San Antonio, TX, November 2001.

[9] M.M. Mansour and N.R. Shanbhag. High-throughput LDPC decoders. IEEE Trans. Very Large Scale Integration (VLSI) Systems, 11:976-996, December 2003.

[10] H. Kfir and I. Kanter. Parallel versus sequential updating for belief propagation decoding. Physica A, 330:259-270, 2003.

[11] J. Zhang and M. Fossorier. Shuffled belief propagation decoding. IEEE Trans. on Commun., 53:209-213, February 2005.

[12] D. Hocevar. A reduced complexity decoder architecture via layered decoding of LDPC codes. In Proc. Signal Processing Systems SIPS 2004, pages 107-112, October 2004.

[13] E. Sharon and S. Litsyn and J. Goldberger. An efficient messagepassing schedule for LDPC decoding. In Proc. 23rd IEEE Convention of Electrical and Electronics Engineers in Israel, pages 223-226, September 2004.
[14] P. Radosavljevic, A. de Baynast, and J. R. Cavallaro. Optimized message-passing schedules for LDPC decoding. In Proc. ThirtyNinth Asilomar Conference on Signals, Systems and Computers, pages 591-595, 2005.

[15] A. I. Vila Casado, M. Griot, and R. D. Wesel. Informed dynamic scheduling for belief-propagation decoding of LDPC codes. In IEEE ICC 2007, pages 932-937, Glasgow, Scotland, July 2007.

[16] P. Roberston, E. Villebrun, and P. Hoeher. A comparison of optimal and sub-optimal MAP decoding algorithm operating in the log domain. In Proc. IEEE Int. Conf. Communications, pages 1009-1013, 1995.

[17] C Jones, S. Dolinar, K. Andrews, D. Divsalar, Y. Zhang, and W. Ryan,. Functions and Architectures for LDPC Decoding. In IEEE ITW 2007, pages 577-583, Lake Tahoe, California, September 2007.

[18] X.Y. Hu, E. Eleftheriou, D.M. Arnold, and A. Dholakia. Efficient Implementations of the Sum-Product Algorithm for Decoding LDPC Codes. In Proc. IEEE Global Conference on Communications, pages 1036-1036E, San Antonio, TX, November 2001.

[19] L. R. Bahl, J. Cocke, F. Jelinek, and J. Raviv. Optimal Decoding of Linear Codes for Minimizing Symbol Error Rate. IEEE Trans. Info. Theory, IT-20:284-287, March 1974.

[20] Wireless LAN Medium Access Control (MAC) and Physical Layer (PHY) specifications, IEEE std. 802.11a, 1999. 